\title{
Analyses of In-cage Singlet Radical-pair Motions from Irradiations of 1-Naphthyl $(R)$-1-Phenylethyl Ether and 1-Naphthyl (R)-2-Phenylpropanoate in $n$-Alkanes
}

\author{
Jinqi Xu and Richard G. Weiss* \\ Department of Chemistry, Georgetown University, Washington, DC 20057-1227, USA
}

\section{Table of Contents}

page

$\begin{array}{lr}\text { Figure } \mathrm{S} 1 . E e \% \text { and } \mathbf{2 B N} / \mathbf{4 B N} \text { ratios versus \%conversion } & \mathrm{S} 2\end{array}$

Table S1. Product distribution from irradiations of $(R)-\mathbf{1} \quad$ S2

Tables S2-S5. Distribution and $e e^{\%}$ of products from irradiations of $(R)-2$ in hexane S2-4

Figures S2-S3. Data extrapolation to 0\% conversion of $(R)-2$ through linear regressions $\quad$ S3-4

Tables S6-S9. Distribution and $e e^{\%}$ of products from irradiations of $(R)-2$ in decane S5-6

Tables S10-S13. Distribution and $e e \%$ of products from irradiations of $(R)-2$ in tetradecane S6-8

Tables S14-S15. Distribution and $e e \%$ of products

from irradiations of $(R)$-2 in heptadecane $\quad$ S8-9

Eqs S1-S14. Calculation of in-cage relative product yields in Table $1 \quad$ S9-11

Table S16. Sum of relative product yields from photoreactions in $n$-alkanes S11

Table S17. Microviscosities of liquid $n$-alkanes calculated from equation in ref. $40 \quad \mathrm{~S} 12$

Eqs S15-S28. Derivation of $\mathrm{P}_{\text {inv }}, \mathrm{P}_{\text {ret, }}$, and $\mathbf{S}$ from Scheme $2 \quad$ S12-13

$\begin{array}{lr}\text { Eqs S29-S31. Derivation of eq } 5 \text { from Scheme } 4 & \text { S13-14 }\end{array}$

Figure S4. Plots of product ratios and ee versus $\ln \left(1 / \eta_{\mathrm{m}}\right)$ for irradiations of $(R)-2 \quad \mathrm{~S} 14$

Eqs S32-S34 and Table S18. Derivation of equations for and calculation

of $(S) /(R)$ enantiomer ratios 


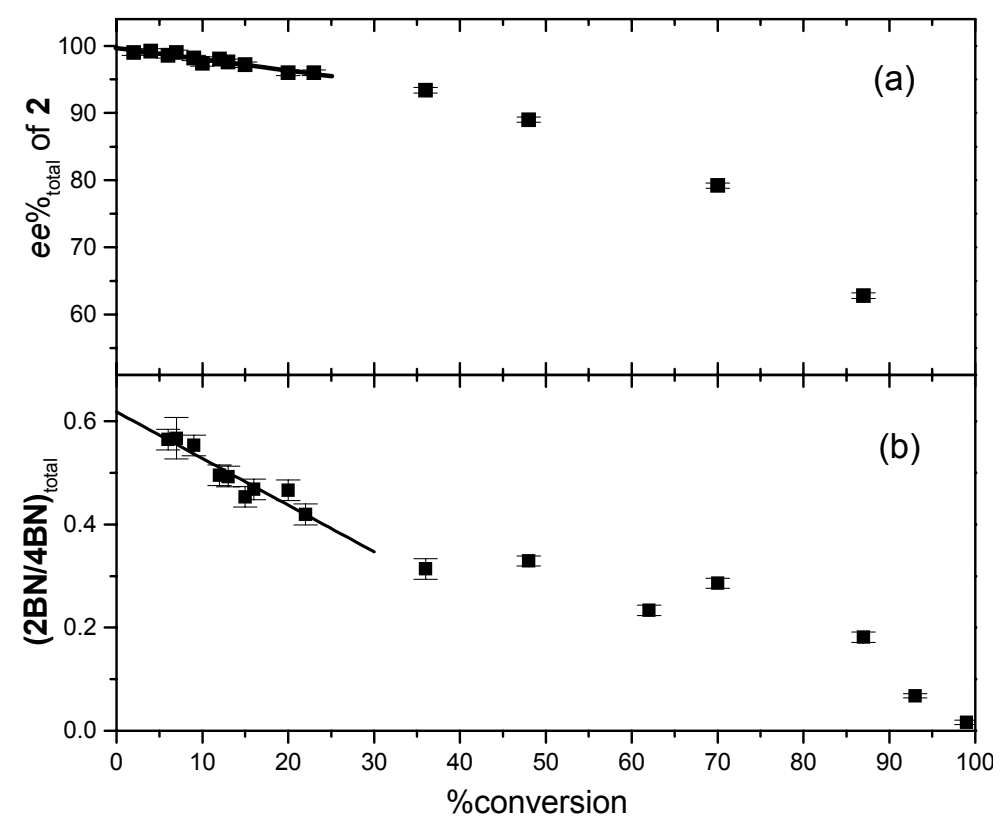

Figure S1. $E e^{\%} \%_{\text {total }}$ of 2 (a) and $(\mathbf{2 B N} / \mathbf{4 B N})_{\text {total }}$ ratios (b) versus $\%$ conversion of $(R)-2$ in hexane at 23 ${ }^{\circ} \mathrm{C}$. The straight lines are best fits to data points below ca. 30\% conversions of $(R)-2$ and they provide the values extrapolated to $0 \%$ conversion used within the text.

Table S1. Product distributions from irradiations of $(R)-1$ in hexane at $23{ }^{\circ} \mathrm{C}^{a}$

\begin{tabular}{ccccccccc}
\hline $\mathbf{2 A N}$ & $\mathbf{4 A N}$ & $\mathbf{2}$ & $\mathbf{2 B N}$ & $\mathbf{4 B N}$ & $\mathbf{3}$ & $\mathbf{5}$ & $\mathbf{4}$ & $\mathbf{6}$ \\
\hline $43.0 \pm 2.7$ & $22.8 \pm 2.0$ & $3.1 \pm 0.1$ & $4.3 \pm 0.3$ & $12.3 \pm 0.5$ & $4.5 \pm 0.4$ & $2.5 \pm 0.8$ & 0 & $7.5 \pm 2.9$
\end{tabular}

${ }^{a}$ Relative yield (\%); the sum of all products is taken as $100 \%$; conversions of $(R)-1$ were always $<$ $30 \%$; mass balance is $>80 \%$.

Table S2. Product distributions from irradiations of $(R)-2$ in hexane at $23{ }^{\circ} \mathrm{C}^{a}$

\begin{tabular}{cccccccc}
\hline \% Conversion of $(R)-\mathbf{2}$ & $\mathbf{2 B N}$ & $\mathbf{4 B N}$ & $\mathbf{3}$ & $\mathbf{5}$ & $\mathbf{6}$ & $(\mathbf{2 B N} / \mathbf{4 B N})_{\text {total }}$ & $((S)-\mathbf{2} /(\mathbf{2 B N}+\mathbf{4 B N}))_{\text {total }}$ \\
\hline 6 & $26.7 \pm 1.0$ & $47.3 \pm 1.0$ & $10.3 \pm 1.0$ & $10.5 \pm 0.5$ & $5.2 \pm 0.5$ & $0.56 \pm 0.02$ & $0.14 \pm 0.02$ \\
\hline 7 & $27.2 \pm 3.5$ & $48.0 \pm 1.8$ & $8.9 \pm 1.0$ & $10.6 \pm 1.0$ & $5.3 \pm 1.0$ & $0.57 \pm 0.06$ & $0.10 \pm 0.02$ \\
\hline 9 & $26.5 \pm 1.1$ & $47.9 \pm 1.1$ & $9.8 \pm 1.0$ & $11.5 \pm 0.5$ & $4.3 \pm 0.5$ & $0.55 \pm 0.03$ & $0.12 \pm 0.01$ \\
\hline 12 & $24.4 \pm 1.2$ & $49.4 \pm 1.0$ & $10.5 \pm 1.0$ & $10.9 \pm 1.0$ & $4.8 \pm 0.5$ & $0.50 \pm 0.03$ & $0.10 \pm 0.01$ \\
\hline 13 & $24.9 \pm 0.8$ & $50.6 \pm 1.0$ & $10.4 \pm 0.5$ & $9.6 \pm 0.5$ & $4.5 \pm 0.5$ & $0.49 \pm 0.02$ & $0.11 \pm 0.01$ \\
\hline 15 & $23.5 \pm 0.6$ & $51.9 \pm 1.0$ & $10.4 \pm 0.6$ & $9.8 \pm 0.5$ & $4.4 \pm 0.5$ & $0.45 \pm 0.02$ & $0.10 \pm 0.01$ \\
\hline
\end{tabular}




\begin{tabular}{|c|c|c|c|c|c|c|c|}
\hline 16 & $22.7 \pm 1.0$ & $48.5 \pm 2.5$ & $8.7 \pm 0.6$ & $11.1 \pm 0.5$ & $9.0 \pm 2.2$ & $0.47 \pm 0.03$ & $0.10 \pm 0.01$ \\
\hline 20 & $24.2 \pm 0.5$ & $52.0 \pm 1.0$ & $8.7 \pm 0.5$ & $10.3 \pm 0.5$ & $4.8 \pm 0.5$ & $0.47 \pm 0.02$ & $0.11 \pm 0.01$ \\
\hline 22 & $22.1 \pm 1.0$ & $52.7 \pm 1.6$ & $9.0 \pm 1.0$ & $10.3 \pm 0.5$ & $5.9 \pm 0.5$ & $0.42 \pm 0.03$ & $0.10 \pm 0.01$ \\
\hline 36 & $17.1 \pm 0.5$ & $54.3 \pm 1.0$ & $12.4 \pm 0.5$ & $10.6 \pm 0.5$ & $5.6 \pm 0.5$ & $0.31 \pm 0.02$ & $0.09 \pm 0.01$ \\
\hline 48 & $18.5 \pm 1.0$ & $56.0 \pm 1.0$ & $9.4 \pm 0.5$ & $9.9 \pm 0.5$ & $6.2 \pm 0.5$ & $0.33 \pm 0.02$ & $0.08 \pm 0.01$ \\
\hline
\end{tabular}

${ }^{a}$ Relative yield (\%); the sum of all products is taken as $100 \%$; mass balance is $>80 \%$.

Table S3. $E e \%$ total of photoproducts from irradiations of $(R)-2$ in hexane at $23{ }^{\circ} \mathrm{C} .{ }^{a}$

\begin{tabular}{cccc}
\hline \multirow{2}{*}{ \% Conversion of $(R)$-2 } & \multicolumn{3}{c}{$e e^{o}$ ototal $_{\text {to }}$} \\
& remaining $\mathbf{2}^{b}$ & 2BN & 4BN \\
\hline 2 & 99.0 & 29.4 & 18.8 \\
\hline 4 & 99.2 & 31.3 & 17.4 \\
\hline 6 & 98.6 & 28.6 & 17.2 \\
\hline 7 & 99.0 & 27.3 & 17.8 \\
\hline 9 & 98.2 & 27.6 & 17.6 \\
\hline 10 & 97.4 & 27.4 & 18.8 \\
\hline 12 & 98.0 & 27.4 & 17.2 \\
\hline 13 & 97.6 & 26.6 & 16.8 \\
\hline 15 & 97.2 & 25.6 & 16.0 \\
\hline 20 & 96.0 & 24.8 & 16.0 \\
\hline 23 & 96.0 & 24.2 & 17.2 \\
\hline 36 & 93.4 & 25.4 & 15.6 \\
\hline 48 & 89.0 & 22.6 & 12.2 \\
\hline
\end{tabular}

${ }^{a} \pm 1.0 \%$; ee of $(R)-2$ before irradiation is $99.5 \% .^{b}(R)$-enantiomer in excess.

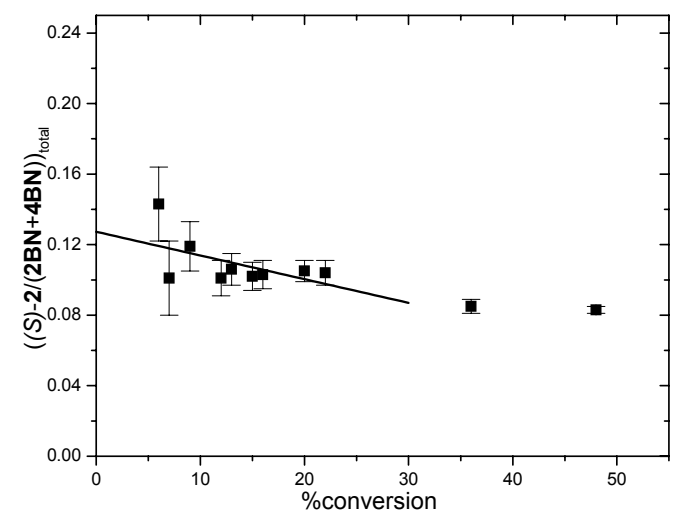

Figure S2. Plot of $((S)-\mathbf{2} /(\mathbf{2 B N}+\mathbf{4 B N}))_{\text {total }}$ versus $\%$ conversion for irradiations of $(R)-2$ in hexane at 23 ${ }^{\circ} \mathrm{C}$. 

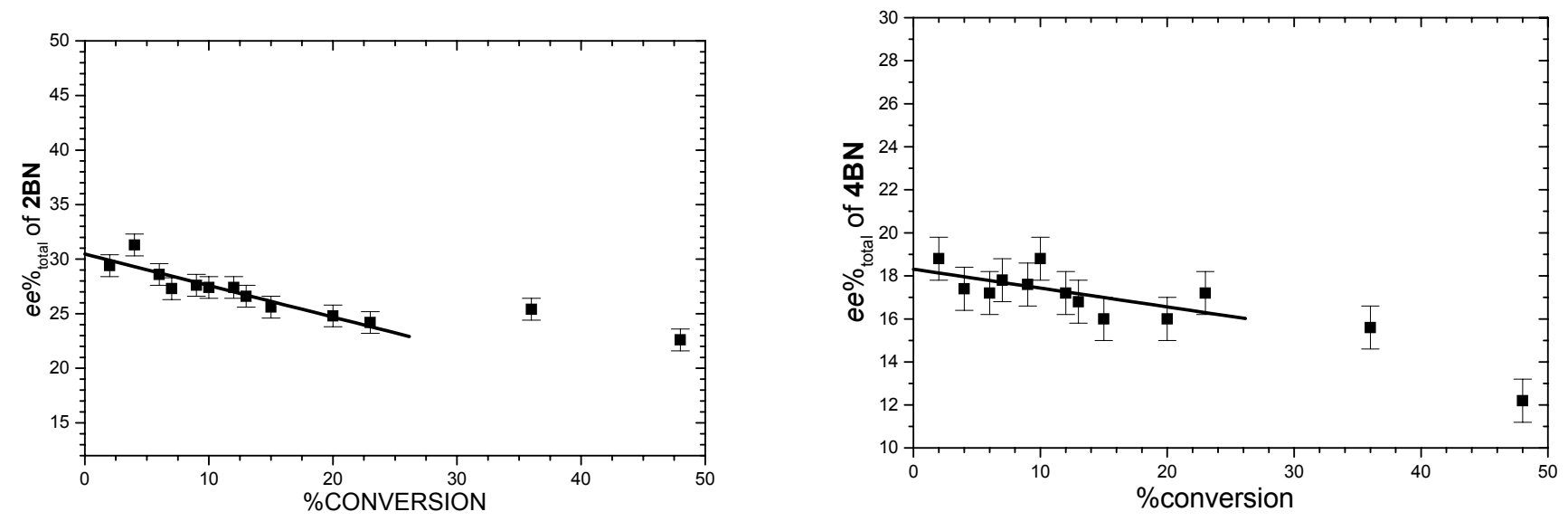

Figure S3. $E e \%_{\text {total }}$ of $\mathbf{2 B N}$ (left) and $4 \mathbf{B N}$ (right) versus $\%$ conversion for irradiations of $(R)-2$ in hexane at $23{ }^{\circ} \mathrm{C}$.

Table S4. Product distributions from irradiations of $(R)-2$ in hexane at $60{ }^{\circ} \mathrm{C} .{ }^{a}$

\begin{tabular}{cccccccc}
\hline \% Conversion of $(R)-\mathbf{2}$ & $\mathbf{2 B N}$ & $\mathbf{4 B N}$ & $\mathbf{3}$ & $\mathbf{5}$ & $\mathbf{6}$ & $(\mathbf{2 B N} / \mathbf{4 B N})_{\text {total }}$ & $((S)-\mathbf{2} /(\mathbf{2 B N}+\mathbf{4 B N}))_{\text {total }}$ \\
\hline 4 & $24.9 \pm 3.0$ & $45.0 \pm 1.0$ & $10.6 \pm 1.5$ & $12.7 \pm 1.0$ & $6.8 \pm 1.0$ & $0.55 \pm 0.06$ & $0.12 \pm 0.04$ \\
\hline 7 & $23.7 \pm 1.8$ & $44.5 \pm 1.0$ & $11.6 \pm 1.5$ & $12.7 \pm 1.0$ & $7.5 \pm 1.0$ & $0.53 \pm 0.04$ & $0.12 \pm 0.02$ \\
\hline 8 & $23.6 \pm 1.0$ & $45.7 \pm 1.2$ & $10.5 \pm 0.5$ & $14.2 \pm 0.5$ & $6.0 \pm 0.5$ & $0.51 \pm 0.03$ & $0.15 \pm 0.02$ \\
\hline 14 & $20.9 \pm 0.7$ & $47.9 \pm 1.1$ & $13.5 \pm 0.7$ & $13.4 \pm 0.5$ & $4.3 \pm 0.5$ & $0.44 \pm 0.02$ & $0.13 \pm 0.01$ \\
\hline 16 & $21.9 \pm 0.6$ & $49.1 \pm 0.6$ & $11.0 \pm 0.5$ & $12.7 \pm 0.5$ & $5.3 \pm 0.5$ & $0.45 \pm 0.02$ & $0.11 \pm 0.01$ \\
\hline 18 & $19.0 \pm 0.4$ & $51.7 \pm 1.0$ & $10.7 \pm 0.5$ & $12.2 \pm 0.5$ & $6.4 \pm 0.5$ & $0.37 \pm 0.02$ & $0.10 \pm 0.01$ \\
\hline 23 & $18.3 \pm 1.5$ & $51.3 \pm 1.0$ & $10.3 \pm 0.5$ & $12.2 \pm 0.5$ & $7.9 \pm 0.5$ & $0.36 \pm 0.03$ & $0.10 \pm 0.01$ \\
\hline 29 & $19.1 \pm 0.5$ & $51.4 \pm 0.5$ & $11.7 \pm 0.5$ & $12.7 \pm 0.5$ & $5.1 \pm 0.5$ & $0.37 \pm 0.02$ & $0.10 \pm 0.01$ \\
\hline 37 & $17.9 \pm 0.5$ & $52.1 \pm 0.6$ & $11.3 \pm 0.9$ & $11.4 \pm 0.5$ & $7.3 \pm 0.5$ & $0.34 \pm 0.02$ & $0.08 \pm 0.01$ \\
\hline 39 & $15.5 \pm 0.5$ & $54.1 \pm 0.5$ & $11.5 \pm 0.5$ & $11.9 \pm 0.5$ & $7.0 \pm 0.5$ & $0.29 \pm 0.02$ & $0.09 \pm 0.01$ \\
\hline
\end{tabular}

${ }^{a}$ Relative yield (\%); the sum of all products is taken as $100 \%$; mass balance is $>80 \%$.

Table S5. $E e \%$ total of photoproducts from irradiations of $(R)-2$ in hexane at $60{ }^{\circ} \mathrm{C} .{ }^{a}$

\begin{tabular}{cccc}
\hline \% Conversion of $(R)-\mathbf{2}$ & \multicolumn{3}{c}{$e \%^{\%}$ total } \\
& remaining $\mathbf{2}^{b}$ & 2BN & 4BN \\
\hline 3 & 99.0 & 26.8 & 15.8 \\
\hline 4 & 99.4 & 26.3 & 14.6 \\
\hline 5 & 98.8 & 26.4 & 15.6 \\
\hline 7 & 98.8 & 24.9 & 14.4 \\
\hline 8 & 98.2 & 23.8 & 15.4 \\
\hline 14 & 97.0 & 24.6 & 16.6 \\
\hline 15 & 97.4 & 23.4 & 13.4 \\
\hline 18 & 97.0 & 22.0 & 13.2 \\
\hline 23 & 95.8 & 22.6 & 13.2
\end{tabular}




\begin{tabular}{cccc}
\hline 24 & 95.6 & 21.9 & 14.8 \\
\hline 29 & 94.4 & 19.4 & 13.2 \\
\hline 37 & 93.4 & 18.0 & 12.2 \\
\hline 39 & 92.2 & 18.6 & 13.0
\end{tabular}

${ }^{\mathrm{a}} \pm 1.0 \%$; ee of $(R)-2$ before irradiation is $99.5 \%{ }^{\mathrm{b}}(R)$-enantiomer in excess.

Table S6. Product distributions from irradiations of $(R)-2$ in decane at $23{ }^{\circ} \mathrm{C} .{ }^{a}$

\begin{tabular}{cccccccc}
\hline \% Conversion of $(R)-\mathbf{2}$ & $\mathbf{2 B N}$ & $\mathbf{4 B N}$ & $\mathbf{3}$ & $\mathbf{5}$ & $\mathbf{6}$ & $(\mathbf{2 B N} / \mathbf{4 B N})_{\text {total }}$ & $((S)-\mathbf{2} /(\mathbf{2 B N}+\mathbf{4 B N}))_{\text {total }}$ \\
\hline 2 & $30.7 \pm 1.3$ & $40.6 \pm 4.0$ & $11.8 \pm 1.8$ & $14.7 \pm 1.0$ & $2.2 \pm 1.0$ & $0.76 \pm 0.08$ & $0.18 \pm 0.06$ \\
\hline 4 & $30.7 \pm 1.4$ & $41.5 \pm 1.0$ & $13.4 \pm 2.4$ & $12.9 \pm 1.0$ & $1.5 \pm 1.0$ & $0.74 \pm 0.04$ & $0.16 \pm 0.04$ \\
\hline 4 & $29.3 \pm 1.2$ & $42.7 \pm 3.5$ & $11.2 \pm 1.5$ & $14.1 \pm 1.0$ & $2.9 \pm 1.0$ & $0.69 \pm 0.06$ & $0.2 \pm 0.05$ \\
\hline 8 & $30.4 \pm 1.3$ & $44.7 \pm 1.0$ & $9.8 \pm 0.5$ & $11.9 \pm 0.5$ & $3.1 \pm 0.5$ & $0.68 \pm 0.05$ & $0.16 \pm 0.02$ \\
\hline 9 & $29.9 \pm 1.5$ & $45.5 \pm 1.0$ & $9.6 \pm 1.7$ & $11.5 \pm 1.5$ & $3.4 \pm 1.0$ & $0.66 \pm 0.04$ & $0.14 \pm 0.02$ \\
\hline 10 & $28.3 \pm 1.0$ & $43.8 \pm 1.0$ & $9.0 \pm 0.5$ & $16.5 \pm 0.5$ & $2.5 \pm 0.5$ & $0.64 \pm 0.03$ & $0.13 \pm 0.02$ \\
\hline 15 & $28.8 \pm 0.6$ & $46.3 \pm 2.0$ & $10.4 \pm 0.6$ & $11.1 \pm 1.0$ & $3.4 \pm 0.5$ & $0.62 \pm 0.03$ & $0.11 \pm 0.01$ \\
\hline 20 & $25.9 \pm 0.5$ & $50.1 \pm 0.5$ & $9.1 \pm 0.5$ & $11.4 \pm 0.5$ & $3.5 \pm 0.5$ & $0.52 \pm 0.02$ & $0.09 \pm 0.01$ \\
\hline 24 & $25.1 \pm 0.5$ & $50.9 \pm 0.5$ & $10.1 \pm 0.6$ & $11.1 \pm 0.5$ & $2.8 \pm 0.5$ & $0.49 \pm 0.02$ & $0.09 \pm 0.01$ \\
\hline 25 & $23.1 \pm 0.5$ & $51.2 \pm 0.5$ & $11.6 \pm 0.5$ & $10.9 \pm 0.5$ & $3.3 \pm 0.5$ & $0.45 \pm 0.02$ & $0.09 \pm 0.01$ \\
\hline 33 & $23.0 \pm 0.6$ & $52.0 \pm 0.5$ & $10.9 \pm 1.2$ & $10.7 \pm 1.0$ & $3.4 \pm 0.5$ & $0.44 \pm 0.02$ & $0.09 \pm 0.01$ \\
\hline 35 & $23.4 \pm 0.5$ & $52.7 \pm 0.5$ & $9.6 \pm 0.5$ & $10.9 \pm 0.5$ & $3.4 \pm 0.5$ & $0.44 \pm 0.02$ & $0.08 \pm 0.01$ \\
\hline 37 & $23.5 \pm 0.4$ & $52.2 \pm 0.5$ & $10.9 \pm 0.6$ & $10.8 \pm 0.5$ & $2.7 \pm 0.5$ & $0.45 \pm 0.02$ & $0.06 \pm 0.01$ \\
\hline
\end{tabular}

${ }^{a}$ Relative yield (\%); the sum of all products is taken as $100 \%$; mass balance is $>80 \%$.

Table S7. Ee\% $\%_{\text {total }}$ of photoproducts from irradiations of (R)-2 in decane at $23{ }^{\circ} \mathrm{C} .{ }^{a}$

\begin{tabular}{cccc}
\hline \multirow{2}{*}{ \% Conversion of $(R)-\mathbf{2}$} & \multicolumn{3}{c}{$e \%^{\%}{ }_{\text {total }}$} \\
& remaining $\mathbf{2}^{b}$ & 2BN & 4BN \\
\hline 3 & 99.0 & 38.2 & 21.2 \\
\hline 4 & 99.0 & 37.9 & 22.6 \\
\hline 7 & 98.4 & 36.6 & 21.2 \\
\hline 8 & 98.2 & 36.6 & 21.6 \\
\hline 9 & 98.0 & 37.1 & 21.2 \\
\hline 10 & 98.0 & 37.0 & 21.0 \\
\hline 15 & 97.2 & 34.6 & 18.9 \\
\hline 20 & 96.4 & 32.4 & 15.3 \\
\hline 24 & 95.8 & 31.2 & 16.4 \\
\hline 25 & 95.8 & 32.0 & 16.8 \\
\hline 33 & 93.8 & 29.6 & 16.5 \\
\hline 35 & 93.2 & 30.6 & 14.4 \\
\hline 37 & 94.6 & 30.8 & 13.4 \\
\hline
\end{tabular}

${ }^{a} \pm 1.0 \%$; ee of $(R)-2$ before irradiation is $99.5 \% .^{b}(R)$-enantiomer in excess. 
Table S8. Product distributions from irradiations of $(R)-2$ in decane at $60{ }^{\circ} \mathrm{C} .{ }^{a}$

\begin{tabular}{cccccccc}
\hline \% Conversion of $(R)-\mathbf{2}$ & $\mathbf{2 B N}$ & $\mathbf{4 B N}$ & $\mathbf{3}$ & $\mathbf{5}$ & $\mathbf{6}$ & $(\mathbf{2 B N} / \mathbf{4 B N})_{\text {total }}$ & $((S)-\mathbf{2} /(\mathbf{2 B N}+\mathbf{4 B N}))_{\text {total }}$ \\
\hline 3 & $26.3 \pm 2.1$ & $42.8 \pm 2.1$ & $9.8 \pm 1.2$ & $18.5 \pm 1.0$ & $2.6 \pm 1.0$ & $0.61 \pm 0.04$ & $0.15 \pm 0.04$ \\
\hline 4 & $30.0 \pm 1.2$ & $49.1 \pm 1.2$ & $12.3 \pm 1.2$ & $7.2 \pm 1.0$ & $1.5 \pm 1.0$ & $0.61 \pm 0.04$ & $0.12 \pm 0.02$ \\
\hline 7 & $28.0 \pm 0.5$ & $48.5 \pm 1.0$ & $11.9 \pm 0.7$ & $9.7 \pm 1.0$ & $1.8 \pm 1.0$ & $0.58 \pm 0.04$ & $0.12 \pm 0.02$ \\
\hline 9 & $25.8 \pm 1.0$ & $47.5 \pm 1.0$ & $12.4 \pm 1.0$ & $11.6 \pm 1.0$ & $2.6 \pm 1.0$ & $0.54 \pm 0.05$ & $0.12 \pm 0.02$ \\
\hline 12 & $23.9 \pm 1.9$ & $46.0 \pm 3.0$ & $13.1 \pm 1.3$ & $14.0 \pm 1.0$ & $3.0 \pm 1.0$ & $0.52 \pm 0.05$ & $0.12 \pm 0.01$ \\
\hline 15 & $22.1 \pm 0.7$ & $50.6 \pm 1.3$ & $12.7 \pm 1.3$ & $12.4 \pm 0.5$ & $2.2 \pm 0.5$ & $0.44 \pm 0.02$ & $0.10 \pm 0.01$ \\
\hline 18 & $21.6 \pm 0.6$ & $48.8 \pm 1.1$ & $13.3 \pm 0.6$ & $13.7 \pm 0.5$ & $2.6 \pm 1.0$ & $0.44 \pm 0.02$ & $0.10 \pm 0.01$ \\
\hline 23 & $20.3 \pm 0.5$ & $51.2 \pm 0.5$ & $13.3 \pm 1.0$ & $12.7 \pm 0.5$ & $2.6 \pm 0.5$ & $0.40 \pm 0.02$ & $0.09 \pm 0.01$ \\
\hline 24 & $19.2 \pm 0.5$ & $51.0 \pm 0.8$ & $13.9 \pm 0.5$ & $12.9 \pm 0.5$ & $2.9 \pm 0.5$ & $0.38 \pm 0.02$ & $0.09 \pm 0.01$
\end{tabular}

${ }^{a}$ Relative yield (\%); the sum of all products is taken as $100 \%$; mass balance is $>80 \%$.

Table S9. $E e^{\%} \%_{\text {total }}$ of photoproducts from irradiations of $(R)-2$ in decane at $60{ }^{\circ} \mathrm{C} .{ }^{a}$

\begin{tabular}{cccc}
\hline \% Conversion of $(R)-\mathbf{2}$ & \multicolumn{3}{c}{$e \%_{\text {total }}$} \\
& ${\text { remaining } \mathbf{2}^{b}}^{b}$ & $\mathbf{2 B N}$ & 4BN \\
\hline 4 & 99.0 & 35.4 & 21.8 \\
\hline 7 & 98.6 & 34.8 & 21.0 \\
\hline 9 & 98.2 & 31.4 & 19.8 \\
\hline 15 & 97.0 & 29.0 & 18.8 \\
\hline 18 & 97.0 & 32.0 & 22.0 \\
\hline 23 & 95.8 & 27.9 & 18.4 \\
\hline 24 & 96.0 & 28.4 & 18.7 \\
\hline 28 & 95.4 & 27.6 & 19.0 \\
\hline
\end{tabular}

${ }^{a} \pm 1.0 \%$; ee of $(R)-2$ before irradiation is $99.5 \%{ }^{b}(R)$-enantiomer in excess.

Table S10. Product distributions from irradiations of $(R)-2$ in tetradecane at $23{ }^{\circ} \mathrm{C} .{ }^{a}$

\begin{tabular}{cccccccc}
\hline \% Conversion of $(R)-\mathbf{2}$ & $\mathbf{2 B N}$ & $\mathbf{4 B N}$ & $\mathbf{3}$ & $\mathbf{5}$ & $\mathbf{6}$ & $\mathbf{( 2 B N / 4 B N})_{\text {total }}$ & $((S)-\mathbf{2} /(\mathbf{2 B N}+\mathbf{4 B N}))_{\text {total }}$ \\
\hline 3 & $34.1 \pm 2.2$ & $41.6 \pm 1.7$ & $11.1 \pm 1.7$ & $13.2 \pm 1.0$ & $b$ & $0.82 \pm 0.06$ & $0.13 \pm 0.04$ \\
\hline 4 & $37.8 \pm 1.2$ & $44.4 \pm 1.7$ & $12.8 \pm 1.9$ & $5.0 \pm 1.0$ & $b$ & $0.85 \pm 0.04$ & $0.17 \pm 0.03$ \\
\hline 5 & $32.8 \pm 1.1$ & $44.2 \pm 3.0$ & $12.6 \pm 1.1$ & $10.4 \pm 1.0$ & $b$ & $0.74 \pm 0.06$ & $0.12 \pm 0.03$ \\
\hline 5 & $36.6 \pm 2.0$ & $42.4 \pm 2.0$ & $10.3 \pm 1.0$ & $10.7 \pm 1.0$ & $b$ & $0.86 \pm 0.06$ & $0.12 \pm 0.03$ \\
\hline 8 & $32.9 \pm 0.6$ & $45.6 \pm 1.4$ & $10.1 \pm 1.3$ & $11.5 \pm 0.5$ & $b$ & $0.72 \pm 0.03$ & $0.14 \pm 0.02$ \\
\hline 9 & $35.3 \pm 0.6$ & $47.5 \pm 0.6$ & $12.9 \pm 1.2$ & $4.3 \pm 0.5$ & $b$ & $0.74 \pm 0.03$ & $0.12 \pm 0.02$ \\
\hline 10 & $33.2 \pm 1.0$ & $49.1 \pm 0.5$ & $13.5 \pm 1.1$ & $4.3 \pm 0.5$ & $b$ & $0.68 \pm 0.03$ & $0.11 \pm 0.02$ \\
\hline 14 & $31.4 \pm 0.5$ & $46.5 \pm 0.5$ & $13.3 \pm 0.5$ & $8.9 \pm 0.5$ & $b$ & $0.68 \pm 0.02$ & $0.11 \pm 0.01$ \\
\hline 15 & $29.4 \pm 0.8$ & $49.2 \pm 0.5$ & $15.7 \pm 0.7$ & $5.8 \pm 0.5$ & $b$ & $0.60 \pm 0.02$ & $0.10 \pm 0.01$ \\
\hline
\end{tabular}




\begin{tabular}{cccccccc}
\hline 16 & $29.6 \pm 1.0$ & $47.0 \pm 1.6$ & $11.7 \pm 1.8$ & $11.7 \pm 1.0$ & $b$ & $0.63 \pm 0.03$ & $0.10 \pm 0.01$ \\
\hline 18 & $28.6 \pm 0.6$ & $47.7 \pm 1.4$ & $12.3 \pm 1.0$ & $11.4 \pm 0.5$ & $b$ & $0.60 \pm 0.02$ & $0.09 \pm 0.01$ \\
\hline 21 & $27.5 \pm 0.7$ & $49.7 \pm 0.5$ & $14.0 \pm 0.5$ & $8.8 \pm 0.5$ & $b$ & $0.55 \pm 0.02$ & $0.09 \pm 0.01$ \\
\hline 23 & $25.2 \pm 0.6$ & $48.9 \pm 0.5$ & $13.6 \pm 1.0$ & $12.3 \pm 0.5$ & $b$ & $0.52 \pm 0.02$ & $0.09 \pm 0.01$ \\
\hline 24 & $24.7 \pm 0.5$ & $49.6 \pm 1.0$ & $13.1 \pm 0.5$ & $12.6 \pm 0.5$ & $b$ & $0.50 \pm 0.02$ & $0.09 \pm 0.01$ \\
\hline
\end{tabular}

${ }^{a}$ Relative yield (\%); the sum of all products is taken as $100 \%$; mass balance is $>80 \%$. ${ }^{b}$ The peak of $\mathbf{6}$ was overlapped by other peaks in the GC and HPLC chromatograms; from an interpolation of data from irradiations of $(R)-2$ in decane and heptadecane at $23{ }^{\circ} \mathrm{C}$, the relative yield of $\mathbf{6}$ in tetradecane is estimated to be $<2.4 \%$.

Table S11. $E e \%$ total of products from irradiations of $(R)-2$ in tetradecane at $23{ }^{\circ} \mathrm{C}^{a}$

\begin{tabular}{cccc}
\hline \% Conversion of $(R)-\mathbf{2}$ & \multicolumn{2}{c}{$e \boldsymbol{\%}_{\text {total }}$} & \\
& remaining $\mathbf{2}^{b}$ & $\mathbf{2 B N}$ & $\mathbf{4 B N}$ \\
\hline 3 & & & 30.8 \\
\hline 4 & 99.0 & 47.9 & 30.0 \\
\hline 5 & 98.8 & 46.2 & 31.7 \\
\hline 8 & 99.0 & 47.8 & 31.4 \\
\hline 10 & 98.2 & 44.8 & 29.8 \\
\hline 14 & 98.2 & 44.4 & 30.0 \\
\hline 15 & 97.4 & 42.4 & 29.0 \\
\hline 16 & 97.4 & 42.8 & 27.2 \\
\hline 21 & 97.2 & 43.4 & 29.0 \\
\hline 23 & 97.0 & 42.6 & 28.8 \\
\hline 24 & 96.5 & 41.6 & 27.6 \\
\hline
\end{tabular}

${ }^{a} \pm 1.0 \%$; ee of $(R)-2$ before irradiation is $99.5 \% .^{b}(R)$-enantiomer in excess.

Table S12. Product distributions from irradiations of $(R)-2$ in tetradecane at $60{ }^{\circ} \mathrm{C} .{ }^{a}$

\begin{tabular}{cccccccc}
\hline \% Conversion of $(R)-\mathbf{2}$ & $\mathbf{2 B N}$ & $\mathbf{4 B N}$ & $\mathbf{3}$ & $\mathbf{5}$ & $\mathbf{6}$ & $\mathbf{( 2 B N / 4 B N})_{\text {total }}$ & $((S)-\mathbf{2} /(\mathbf{2 B N}+\mathbf{4 B N}))_{\text {total }}$ \\
\hline 4 & $29.1 \pm 1.8$ & $41.2 \pm 1.3$ & $15.5 \pm 1.8$ & $14.2 \pm 1.0$ & $b$ & $0.71 \pm 0.05$ & $0.14 \pm 0.04$ \\
\hline 5 & $34.2 \pm 1.6$ & $48.4 \pm 1.1$ & $14.7 \pm 1.1$ & $2.7 \pm 1.0$ & $b$ & $0.71 \pm 0.04$ & $0.14 \pm 0.03$ \\
\hline 6 & $30.8 \pm 0.8$ & $49.2 \pm 1.1$ & $15.5 \pm 1.0$ & $4.6 \pm 1.0$ & $b$ & $0.63 \pm 0.03$ & $0.13 \pm 0.03$ \\
\hline 8 & $27.3 \pm 1.3$ & $45.3 \pm 2.2$ & $16.4 \pm 1.3$ & $11.0 \pm 1.0$ & $b$ & $0.60 \pm 0.04$ & $0.13 \pm 0.02$ \\
\hline 9 & $25.7 \pm 1.2$ & $43.2 \pm 1.4$ & $17.9 \pm 1.1$ & $13.2 \pm 1.0$ & $b$ & $0.59 \pm 0.03$ & $0.13 \pm 0.02$ \\
\hline 13 & $22.0 \pm 0.5$ & $47.0 \pm 0.5$ & $18.1 \pm 0.5$ & $12.8 \pm 0.5$ & $b$ & $0.47 \pm 0.02$ & $0.10 \pm 0.01$ \\
\hline 14 & $20.8 \pm 0.8$ & $46.7 \pm 1.2$ & $19.5 \pm 1.4$ & $13.0 \pm 1.0$ & $b$ & $0.45 \pm 0.02$ & $0.12 \pm 0.01$ \\
\hline 20 & $18.6 \pm 0.5$ & $48.1 \pm 0.5$ & $19.7 \pm 0.7$ & $13.6 \pm 0.5$ & $b$ & $0.39 \pm 0.02$ & $0.10 \pm 0.01$ \\
\hline 26 & $15.2 \pm 0.5$ & $50.2 \pm 0.5$ & $20.4 \pm 0.5$ & $14.1 \pm 0.5$ & $b$ & $0.30 \pm 0.02$ & $0.09 \pm 0.01$ \\
\hline
\end{tabular}

${ }^{a}$ Relative yield (\%); the sum of all products is taken as $100 \%$; mass balance is $>80 \%{ }^{b}$ The peak of $\mathbf{6}$ was overlapped with others in the GC and HPLC chromatograms; from irradiations of $(R)-2$ in decane at 60 ${ }^{\circ} \mathrm{C}$, we estimate the yield of 6 to be no more than $2.5 \%$. 
Table S13. $E e \%$ total of products from irradiations of $(R)-2$ in tetradecane at $60{ }^{\circ} \mathrm{C} .{ }^{a}$

\begin{tabular}{cccc}
\hline \multirow{2}{*}{ \% Conversion of $(R)-\mathbf{2}$} & \multicolumn{3}{c}{$e \boldsymbol{\%}_{\text {total }}$} \\
& remaining $\mathbf{2}^{b}$ & $\mathbf{2 B N}$ & 4BN \\
\hline 4 & 99.2 & 40.2 & 28.0 \\
\hline 5 & 98.8 & 40.0 & 26.2 \\
\hline 6 & 98.6 & 39.4 & 24.8 \\
\hline 8 & 98.4 & 38.4 & 24.4 \\
\hline 9 & 98.2 & 36.8 & 23.0 \\
\hline 13 & 97.8 & 35.4 & 21.8 \\
\hline 14 & 97.4 & 36.0 & 21.8 \\
\hline 20 & 96.6 & 35.0 & 21.1 \\
\hline 26 & 95.8 & 34.0 & 20.0 \\
\hline 27 & 95.4 & 33.6 & 19.2 \\
\hline
\end{tabular}

${ }^{a} \pm 1.0 \%$; ee of $(R)-2$ before irradiation is $99.5 \% .^{b}(R)$-enantiomer in excess.

Table S14. Product distributions from irradiations of $(R)-2$ in heptadecane at $23{ }^{\circ} C^{a}$

\begin{tabular}{|c|c|c|c|c|c|c|}
\hline$\%$ Conversion of $(R)-2$ & $2 B N$ & $4 B N$ & 3 & 5 & $(\mathbf{2 B N} / \mathbf{4 B N})_{\text {total }}$ & $((S)-\mathbf{2} /(\mathbf{2} \mathbf{B N}+4 \mathbf{B N}))_{\text {total }}$ \\
\hline 5 & $36.9 \pm 1.6$ & $44.2 \pm 2.8$ & $15.2 \pm 2.4$ & $3.6 \pm 1.0$ & $0.83 \pm 0.06$ & $0.16 \pm 0.03$ \\
\hline 6 & $36.6 \pm 0.9$ & $45.1 \pm 1.8$ & $13.6 \pm 0.9$ & $4.8 \pm 0.5$ & $0.81 \pm 0.03$ & $0.17 \pm 0.03$ \\
\hline 9 & $31.3 \pm 2.0$ & $42.9 \pm 1.2$ & $12.9 \pm 2.2$ & $12.9 \pm 1.0$ & $0.73 \pm 0.05$ & $0.21 \pm 0.03$ \\
\hline 10 & $33.4 \pm 1.1$ & $48.6 \pm 1.6$ & $12.3 \pm 1.1$ & $5.7 \pm 0.5$ & $0.69 \pm 0.03$ & $0.13 \pm 0.01$ \\
\hline 11 & $33.5 \pm 0.5$ & $49.6 \pm 0.5$ & $13.3 \pm 0.5$ & $3.6 \pm 0.5$ & $0.67 \pm 0.02$ & $0.13 \pm 0.01$ \\
\hline 16 & $31.7 \pm 0.6$ & $50.7 \pm 0.6$ & $12.7 \pm 1.0$ & $4.8 \pm 0.5$ & $0.63 \pm 0.02$ & $0.11 \pm 0.01$ \\
\hline 18 & $30.5 \pm 0.5$ & $52.5 \pm 0.5$ & $13.7 \pm 0.5$ & $3.3 \pm 0.5$ & $0.58 \pm 0.02$ & $0.11 \pm 0.01$ \\
\hline 21 & $30.2 \pm 0.5$ & $49.7 \pm 1.1$ & $13.2 \pm 1.0$ & $6.9 \pm 0.5$ & $0.61 \pm 0.02$ & $0.09 \pm 0.01$ \\
\hline 22 & $28.8 \pm 0.6$ & $53.8 \pm 0.5$ & $12.7 \pm 0.5$ & $4.8 \pm 0.5$ & $0.53 \pm 0.02$ & $0.10 \pm 0.01$ \\
\hline 23 & $29.4 \pm 0.5$ & $53.2 \pm 0.5$ & $13.0 \pm 0.5$ & $4.5 \pm 0.5$ & $0.55 \pm 0.02$ & $0.09 \pm 0.01$ \\
\hline 25 & $27.3 \pm 1.4$ & $50.1 \pm 0.6$ & $10.7 \pm 0.5$ & $11.9 \pm 0.5$ & $0.54 \pm 0.03$ & $0.09 \pm 0.01$ \\
\hline
\end{tabular}

${ }^{a}$ Relative yield (\%); the sum of all products is taken as $100 \%$; mass balance is $>80 \%$; the relative yield of 6 was $<1.6 \%$.

Table S15. Ee\% $\%_{\text {total }}$ of products from irradiations of $(R)-2$ in heptadecane at $23{ }^{\circ} \mathrm{C} .{ }^{a}$ 


\begin{tabular}{|c|c|c|c|}
\hline \multirow{2}{*}{$\%$ Conversion of $(R)-\mathbf{2}$} & \multicolumn{3}{|c|}{$e e \%$ total } \\
\hline & remaining $\mathbf{2}^{b}$ & $2 \mathrm{BN}$ & $4 B N$ \\
\hline 4 & 98.6 & 46.0 & 31.1 \\
\hline 5 & 98.6 & 45.4 & 29.2 \\
\hline 6 & 98.4 & 43.5 & 29.8 \\
\hline 9 & 97.2 & 42.8 & 26.8 \\
\hline 10 & 97.8 & 41.8 & 28.0 \\
\hline 11 & 97.4 & 42.5 & 26.5 \\
\hline 16 & 96.6 & 41.1 & 25.8 \\
\hline 18 & 95.8 & 38.9 & 24.6 \\
\hline 21 & 96.4 & 40.4 & 25.8 \\
\hline 23 & 95.4 & 38.2 & 23.6 \\
\hline 25 & 95.5 & 39.0 & 25.2 \\
\hline
\end{tabular}

${ }^{a} \pm 1.0 \%$; ee of $(R)-2$ before irradiation is $99.5 \% .^{b}(R)$-enantiomer in excess.

\section{Calculation of in-cage relative product yields in Table 1.}

We assume: (1) the total amount of out-of-cage radical-pair $\mathbf{B}$ combination products is twice that of 6 ; (2) the $2: \mathbf{2 B N}: 4 \mathbf{B N}$ product ratio from out-of-cage radical-pair $\mathbf{B}$ combinations is constant in liquid $n$-alkane solvents and equal to $1: 1.4: 4.0$, the value when $(R)-\mathbf{1}$ is irradiated in hexane at room temperature (where these products derive from out-of-cage radical-pair B combination; (3) the probability of 1-phenylethyl and 1-naphthoxy radicals with each other is equally probable; the instability of 1-naphthoxy/1-naphthoxy combination products makes this assumption only approximate, at best. Thus,

$((\mathbf{2 B N}+\mathbf{4 B N}) /(\mathbf{2}+\mathbf{2 B N}+\mathbf{4 B N}))_{\text {out-of-cage }}=(1.4+4.0) /(1+1.4+4.0)=5.4 / 6.4$

$(2 /(2+2 B N+4 B N))_{\text {out-of-cage }}=1.0 / 6.4$

Since $(\mathbf{2 B N} / \mathbf{4 B N})_{\text {out-of-cage }}=1.4 / 4.0=0.35$,

$$
\begin{gathered}
(2 \mathrm{BN} /(2 \mathrm{BN}+4 \mathrm{BN}))_{\text {out-of-cage }}=0.35 / 1.35 \\
(4 \mathrm{BN} /(\mathbf{2 B N}+4 \mathrm{BN}))_{\text {out-of-cage }}=1.0 / 1.35
\end{gathered}
$$

The total amounts of $\mathbf{2 B N}$ and $\mathbf{4 B N}$ from out-of-cage combinations are known and $(\mathbf{2} \mathbf{B N}+\mathbf{4 B N})_{\text {out-of- }}$ cage $=2 \times 6 \times[(\mathbf{2 B N}+\mathbf{4 B N}) /(2+\mathbf{2 B N}+\mathbf{4 B N})]_{\text {out-of-cage }}=2 \times 6 \times(5.4 / 6.4)$

So that, $\mathbf{2} \mathbf{B} \mathbf{N}_{\text {in-cage }}=\mathbf{2} \mathbf{B} \mathbf{N}_{\text {total }}-\mathbf{2} \mathbf{B} \mathbf{N}_{\text {out-of-cage }}$ 


$$
\begin{aligned}
& =2 \mathrm{BN}_{\text {total }}-(2 \mathrm{BN}+4 \mathrm{BN})_{\text {out-of-cage }} \times(2 \mathrm{BN} /(2 \mathrm{BN}+4 \mathrm{BN}))_{\text {out-of-cage }} \\
& =\mathbf{2} \mathbf{B} \mathbf{N}_{\text {total }}-2 \times \mathbf{6} \times(5.4 / 6.4) \times(0.35 / 1.35) \\
& 4 B N_{\text {in-cage }}=4 B N_{\text {total }}-4 B N_{\text {out-of-cage }} \\
& =4 \mathrm{BN}_{\text {total }}-(2 \mathrm{BN}+4 \mathrm{BN})_{\text {out-of-cage }} \times(4 \mathrm{BN} /(2 \mathrm{BN}+4 \mathrm{BN}))_{\text {out-of-cage }} \\
& =\mathbf{4 B} \mathbf{N}_{\text {total }}-2 \times 6 \times(5.4 / 6.4) \times(1.0 / 1.35)
\end{aligned}
$$

Then, $(\mathbf{2 B N} / \mathbf{4 B N})_{\text {in-cage }}=\left[\mathbf{2} \mathbf{B N} \mathbf{t o t a l}_{-2} \mathbf{2} \times \mathbf{6} \times(5.4 / 6.4) \times(0.35 / 1.35)\right] /\left[\mathbf{4 B N}_{\text {total }}-2 \times \mathbf{6} \times(5.4 / 6.4) \times(1.0 / 1.35)\right]$

By definition, ee $=([(R)]-[(S)]) /([(R)]+[(S)])$.

Therefore, $1+e e=1+([(R)]-[(S)]) /([(R)]+[(S)])=2[(R)] /([(R)]+[(S)])$

So that, $[(R)] /([(R)]+[(S)])=(1+e e) / 2$

$$
[(S)] /([(R)]+[(S)])=(1-e e) / 2
$$

From $(R)-\mathbf{2} \mathbf{B} \mathbf{N}_{\text {in-cage }}=(R)-\mathbf{2} \mathbf{B} \mathbf{N}_{\text {total }}-(R)-\mathbf{2} \mathbf{B} \mathbf{N}_{\text {out-of-cage, }}(S)-\mathbf{2} \mathbf{B} \mathbf{N}_{\text {in-cage }}=(S)-\mathbf{2} \mathbf{B} \mathbf{N}_{\text {total }}-(S)-\mathbf{2} \mathbf{B} \mathbf{N}_{\text {out-of-cage, }}$ and equations $\mathrm{S} 6$ and $\mathrm{S} 7$,

$$
\begin{aligned}
& (R)-\mathbf{2} \mathbf{B} \mathbf{N}_{\text {in-cage }}=\mathbf{2} \mathbf{B} \mathbf{N}_{\text {total }} \times\left(1+e e_{2 \mathrm{BN} \text {-total }}\right) / 2-\mathbf{2} \mathbf{B} \mathbf{N}_{\text {out-of-cage }} / 2 \\
& (S)-\mathbf{2} \mathbf{B} \mathbf{N}_{\text {in-cage }}=\mathbf{2} \mathbf{B} \mathbf{N}_{\text {total }} \times\left(1-e e_{2 \mathrm{BN} \text {-total }}\right) / 2-\mathbf{2} \mathbf{B} \mathbf{N}_{\text {out-of-cage }} / 2
\end{aligned}
$$

Therefore,

$$
\begin{aligned}
e e_{2 \mathrm{BN} \text {-in-cage }} & =\left((R)-\mathbf{2} \mathbf{B} \mathbf{N}_{\text {in-cage }}-(S)-\mathbf{2} \mathbf{B} \mathbf{N}_{\text {in-cage }}\right) /\left((R)-\mathbf{2} \mathbf{B} \mathbf{N}_{\text {in-cage }}+(S)-\mathbf{2} \mathbf{B} \mathbf{N}_{\text {in-cage }}\right) \\
& =\mathbf{2} \mathbf{B} \mathbf{N}_{\text {total }} \times e e_{2 \mathrm{BN} \text {-total }} /\left(\mathbf{2} \mathbf{B} \mathbf{N}_{\text {total }}-\mathbf{2} \mathbf{B} \mathbf{N}_{\text {out-of-cage }}\right) \\
& =\mathbf{2} \mathbf{B} \mathbf{N}_{\text {total }} \times e e_{2 \mathrm{BN} \text {-total }} / \mathbf{2} \mathbf{B} \mathbf{N}_{\text {in-cage }}
\end{aligned}
$$

It follows by analogy that

$$
e e_{4 \mathrm{BN}-\text { in-cage }}=\mathbf{4} \mathbf{B} \mathbf{N}_{\text {total }} \times e e_{4 \mathrm{BN}-\text { total }} / \mathbf{4} \mathbf{B} \mathbf{N}_{\text {in-cage }}
$$


To obtain the total amount of $\mathbf{2}, \mathbf{2 B N}$, and $\mathbf{4 B N}$ from in-cage radical-pair $\mathbf{B}$ combinations, the $((S)-\mathbf{2} /(\mathbf{2 B N}+\mathbf{4 B N}))_{\text {total }}$ ratio must be employed (see Table S16), and it must be assumed that the lower limit to the amount of $(R)-\mathbf{2}$ generated from in-cage radical-pair $\mathbf{B}$ combination is equal to the amount of $(S)-2$ formed. In addition, it is reasonable to assume that the amount of $(R)-\mathbf{2}$ is equal to the amount of $(S)-2$ formed from out-of-cage combinations. Then,

$$
\begin{aligned}
& \mathbf{2}_{\text {total }}=2 \times((S)-2) \text { total } \\
& =2 \times[(S)-\mathbf{2} /(\mathbf{2} \mathbf{B N}+\mathbf{4 B N})]_{\text {total }} \times(\mathbf{2 B N}+\mathbf{4 B N})_{\text {total }} \\
& (2+2 B N+4 B N)_{\text {in-cage }}=(2 \times(S)-2+2 B N+4 B N)_{\text {in-cage }} \\
& =(2+2 \mathrm{BN}+4 \mathrm{BN})_{\text {total }}-(2+2 \mathrm{BN}+4 \mathrm{BN})_{\text {out-of-cage }} \\
& =(2+2 \mathrm{BN}+4 \mathrm{BN})_{\text {total }}-2 \times 6
\end{aligned}
$$

Since the total of the initial relative product yields (all photoproducts in Table S2 or S4) does not include the regeneration of $\mathbf{2}$, it is necessary to normalize its yield from radical pairs to compare different cases.

After normalization and expressing yields as per cents,

$$
(2 \times(S)-\mathbf{2}+\mathbf{2 B N}+\mathbf{4 B N})_{\text {in-cage }}=100 \times\left((\mathbf{2}+\mathbf{2 B N}+\mathbf{4 B N})_{\text {total }}-2 \times \mathbf{6}\right) /\left(100+\mathbf{2}_{\text {total }}\right)
$$

Table S16. Sum of relative product yields from in-cage radical-pair B combinations of $(R)-2$ in the liquid phases of $\boldsymbol{n}$-alkanes.

\begin{tabular}{cccccc}
\hline Medium & $\mathrm{T}\left({ }^{\circ} \mathrm{C}\right)$ & $\begin{array}{c}((S)-\mathbf{2} / \\
(\mathbf{2 B N}+\mathbf{4 B N}))_{\text {total }}{ }^{a}\end{array}$ & $\begin{array}{c}(\mathbf{2 B N}+\mathbf{4 B N})_{\text {total }}{ }^{a} \\
(2 \times(S)-\mathbf{2}+\mathbf{2 B N}\end{array}$ & $\begin{array}{c}1 /[(2 \times(S)-\mathbf{2}+\mathbf{2 B N} \\
+\mathbf{4 B N})_{\text {in-cage }}\end{array}$ & $+\mathbf{4 B N})_{\text {in-cage }}-1$ \\
\hline Hexane & 23 & 0.13 & 74.7 & 71 & 0.41 \\
Hexane & 60 & 0.14 & 70.6 & 64 & 0.56 \\
Decane & 23 & 0.18 & 73.0 & 74 & 0.35 \\
Decane & 60 & 0.14 & 70.9 & 74 & 0.35 \\
Tetradecane & 23 & 0.15 & 78.9 & 81 & 0.23 \\
Tetradecane & 60 & 0.15 & 76.3 & 78 & 0.28 \\
Heptadecane & 23 & 0.18 & 80.5 & 84 & 0.19 \\
\hline
\end{tabular}

${ }^{a}$ Extrapolated values to $0 \%$ conversion of $(R)-2$. 
Table S17. Microviscosities of liquid $\boldsymbol{n}$-alkanes calculated from equation in ref. 40

\begin{tabular}{ccccccccc}
\hline Molecule & $\begin{array}{c}\mathrm{V} \text { (van der Waals } \\
\left.\text { volume; } \AA^{3}\right)\end{array}$ & $\begin{array}{c}\text { Radius } \\
(\AA)\end{array}$ & $\begin{array}{c}\mathrm{mp} \\
(\mathrm{K})\end{array}$ & $\begin{array}{c}\mathrm{bp} \\
(\mathrm{K})\end{array}$ & $\begin{array}{l}f \text { at } 23{ }^{\circ} \mathrm{C} \\
f \text { at } 60{ }^{\circ} \mathrm{C}\end{array}$ & $\begin{array}{c}\eta_{\mathrm{m}} \text { at } 23^{\circ} \mathrm{C} \\
(\mathrm{cP})\end{array}$ & $\begin{array}{c}\eta_{\mathrm{m}} \text { at } 60{ }^{\circ} \mathrm{C} \\
(\mathrm{cP})\end{array}$ \\
\hline Hexane & 114 & 2.72 & 177.8 & 341.9 & 0.597 & 0.625 & 0.1811 & 0.1389 \\
Decane & 182 & 3.18 & 243.5 & 447.3 & 0.442 & 0.458 & 0.3941 & 0.2527 \\
Tetradecane & 250 & 3.53 & 279.0 & 526.7 & 0.376 & 0.385 & 0.8258 & 0.4443 \\
Heptadecane & 301 & 3.76 & 295.1 & 575.0 & 0.349 & 0.355 & 1.364 & - \\
\hline
\end{tabular}

\section{Derivation of $\mathbf{P}_{\text {inv }}, \mathbf{P}_{\text {ret }}$ and $S$ from Scheme 2.}

Based on Scheme 2, $\mathrm{P}_{\text {ret }}$ and $\mathrm{P}_{\text {inv }}$ for the regeneration of $\mathbf{2}$ and the formation of $\mathbf{2 B N}$ and $\mathbf{4 B N}$ are:

$$
\begin{aligned}
& \mathrm{P}_{\text {ret-2 }}=\mathrm{k}_{2} /\left(\mathrm{k}_{2}+\mathrm{k}_{2 \mathrm{BN}}+\mathrm{k}_{4 \mathrm{BN}}+\mathrm{k}_{\mathrm{t}}+\mathrm{k}_{\mathrm{esc}}\right) \\
& \mathrm{P}_{\text {inv-2 }}=\left[\mathrm{k}_{\mathrm{t}} /\left(\mathrm{k}_{2}+\mathrm{k}_{2 \mathrm{BN}}+\mathrm{k}_{4 \mathrm{BN}}+\mathrm{k}_{\mathrm{t}}+\mathrm{k}_{\mathrm{esc}}\right)\right] \times\left[\mathrm{k}_{2} /\left(\mathrm{k}_{2}+\mathrm{k}_{2 \mathrm{BN}}+\mathrm{k}_{4 \mathrm{BN}}+\mathrm{k}_{\mathrm{t}}+\mathrm{k}_{\mathrm{esc}}\right)\right] \\
& \mathrm{P}_{\text {ret-2BN }}=\mathrm{k}_{2 \mathrm{BN}} /\left(\mathrm{k}_{2}+\mathrm{k}_{2 \mathrm{BN}}+\mathrm{k}_{4 \mathrm{BN}}+\mathrm{k}_{\mathrm{t}}+\mathrm{k}_{\mathrm{esc}}\right) \\
& \mathrm{P}_{\text {inv-2BN }}=\left[\mathrm{k}_{\mathrm{t}} /\left(\mathrm{k}_{2}+\mathrm{k}_{2 \mathrm{BN}}+\mathrm{k}_{4 \mathrm{BN}}+\mathrm{k}_{\mathrm{t}}+\mathrm{k}_{\mathrm{esc}}\right)\right] \times\left[\mathrm{k}_{2 \mathrm{BN}} /\left(\mathrm{k}_{2}+\mathrm{k}_{2 \mathrm{BN}}+\mathrm{k}_{4 \mathrm{BN}}+\mathrm{k}_{\mathrm{t}}+\mathrm{k}_{\mathrm{esc}}\right)\right] \\
& \mathrm{P}_{\text {ret-4BN }}=\mathrm{k}_{4 \mathrm{BNN}} /\left(\mathrm{k}_{2}+\mathrm{k}_{2 \mathrm{BN}}+\mathrm{k}_{4 \mathrm{BN}}+\mathrm{k}_{\mathrm{t}}+\mathrm{k}_{\mathrm{esc}}\right) \\
& \mathrm{P}_{\text {inv-4BN }}=\left[\mathrm{k}_{\mathrm{t}} /\left(\mathrm{k}_{2}+\mathrm{k}_{2 \mathrm{BN}}+\mathrm{k}_{4 \mathrm{BN}}+\mathrm{k}_{\mathrm{t}}+\mathrm{k}_{\mathrm{esc}}\right)\right] \times\left[\mathrm{k}_{4 \mathrm{BN}} /\left(\mathrm{k}_{2}+\mathrm{k}_{2 \mathrm{BN}}+\mathrm{k}_{4 \mathrm{BN}}+\mathrm{k}_{\mathrm{t}}+\mathrm{k}_{\mathrm{esc}}\right)\right]
\end{aligned}
$$

Since

$$
e e=([(R)]-[(S)]) /([(R)]+[(S)])=\left(\mathrm{P}_{\text {ret }}-\mathrm{P}_{\text {inv }}\right) /\left(\mathrm{P}_{\text {ret }}+\mathrm{P}_{\text {inv }}\right)
$$

Therefore,

$$
\begin{aligned}
e e_{2}= & {\left[\mathrm{P}_{\text {ret-2 }}-\mathrm{P}_{\text {inv-2 }}\right] /\left[\mathrm{P}_{\text {ret-2 }}+\mathrm{P}_{\text {inv-2 }}\right] } \\
= & \left(\mathrm{k}_{2}+\mathrm{k}_{2 \mathrm{BN}}+\mathrm{k}_{4 \mathrm{BN}}+\mathrm{k}_{\mathrm{esc}}\right) /\left(\mathrm{k}_{2}+\mathrm{k}_{2 \mathrm{BN}}+\mathrm{k}_{4 \mathrm{BN}}+2 \mathrm{k}_{\mathrm{t}}+\mathrm{k}_{\mathrm{esc}}\right) \\
e e_{2 \mathrm{BN}}= & {\left[\mathrm{P}_{\text {ret-2BN }}-\mathrm{P}_{\text {inv-2BN }}\right] /\left[\mathrm{P}_{\text {ret-2BN }}+\mathrm{P}_{\text {inv-2BN }}\right] } \\
= & {\left[\left(\mathrm{k}_{2}+\mathrm{k}_{2 \mathrm{BN}}+\mathrm{k}_{4 \mathrm{BN}}+\mathrm{k}_{\mathrm{esc}}\right) /\left(\mathrm{k}_{2}+\mathrm{k}_{2 \mathrm{BN}}+\mathrm{k}_{4 \mathrm{BN}}+2 \mathrm{k}_{\mathrm{t}}+\mathrm{k}_{\mathrm{esc}}\right)\right.} \\
e e_{4 \mathrm{BN}}= & {\left[\mathrm{P}_{\text {ret-4BN }}-\mathrm{P}_{\text {inv-4BN}}\right] /\left[\mathrm{P}_{\text {ret-4BN }}+\mathrm{P}_{\text {inv-4BN }}\right] } \\
= & {\left[\left(\mathrm{k}_{2}+\mathrm{k}_{2 \mathrm{BN}}+\mathrm{k}_{4 \mathrm{BN}}+\mathrm{k}_{\mathrm{esc}}\right) /\left(\mathrm{k}_{2}+\mathrm{k}_{2 \mathrm{BN}}+\mathrm{k}_{4 \mathrm{BN}}+2 \mathrm{k}_{\mathrm{t}}+\mathrm{k}_{\mathrm{esc}}\right)\right.}
\end{aligned}
$$


Although eqs S22-S24 predict that the ee values for $\mathbf{2}, \mathbf{2 B N}$, and $\mathbf{4 B N}$ should be the same when extrapolated to $0 \%$ conversion, they clearly are not. This discrepancy demonstrates that even Scheme 2 does not encompass the complexity of all of the radical-pair $\mathbf{B}$ motions. In a still more rigorous treatment, the ensemble average rate constant for tumbling, $\mathrm{k}_{\mathrm{t}}$, must be considered as $\mathrm{k}_{\mathrm{t}-2}+\mathrm{k}_{\mathrm{t}-2 \mathrm{BN}}+\mathrm{k}_{\mathrm{t}}$. $4 \mathrm{BN}$, the sum of the specific tumbling rate constants of 1-phenylethyl radicals that eventually combine with 1-naphthoxy to form each of 2, 2BN, and 4BN.

Therefore, eqs S16, S18, and S20 become:

$$
\begin{aligned}
& \mathrm{P}_{\text {inv-2 }}=\left[\mathrm{k}_{\mathrm{t}-2} /\left(\mathrm{k}_{2}+\mathrm{k}_{2 \mathrm{BN}}+\mathrm{k}_{4 \mathrm{BN}}+\mathrm{k}_{\mathrm{t}}+\mathrm{k}_{\mathrm{esc}}\right)\right] \times\left[\mathrm{k}_{2} /\left(\mathrm{k}_{2}+\mathrm{k}_{2 \mathrm{BN}}+\mathrm{k}_{4 \mathrm{BN}}+\mathrm{k}_{\mathrm{t}}+\mathrm{k}_{\mathrm{esc}}\right)\right] \\
& \mathrm{P}_{\text {inv-2BN }}=\left[\mathrm{k}_{\mathrm{t}-2 \mathrm{BN}} /\left(\mathrm{k}_{2}+\mathrm{k}_{2 \mathrm{BN}}+\mathrm{k}_{4 \mathrm{BN}}+\mathrm{k}_{\mathrm{t}}+\mathrm{k}_{\mathrm{esc}}\right)\right] \times\left[\mathrm{k}_{2 \mathrm{BN}} /\left(\mathrm{k}_{2}+\mathrm{k}_{2 \mathrm{BN}}+\mathrm{k}_{4 \mathrm{BN}}+\mathrm{k}_{\mathrm{t}}+\mathrm{k}_{\mathrm{esc}}\right)\right] \\
& \mathrm{P}_{\text {inv-4BN }}=\left[\mathrm{k}_{\mathrm{t}-4 \mathrm{BN}} /\left(\mathrm{k}_{2}+\mathrm{k}_{2 \mathrm{BN}}+\mathrm{k}_{4 \mathrm{BN}}+\mathrm{k}_{\mathrm{t}}+\mathrm{k}_{\mathrm{esc}}\right)\right] \times\left[\mathrm{k}_{4 \mathrm{BN}} /\left(\mathrm{k}_{2}+\mathrm{k}_{2 \mathrm{BN}}+\mathrm{k}_{4 \mathrm{BN}}+\mathrm{k}_{\mathrm{t}}+\mathrm{k}_{\mathrm{esc}}\right)\right]
\end{aligned}
$$

Based on eqs S15 and S25,

$$
\begin{aligned}
& \mathbf{S}=2 \mathrm{P}_{\mathrm{inv}-2} /\left[1-\mathrm{P}_{\text {ret-2 }}-\mathrm{P}_{\mathrm{inv}-2}\right] \\
& =2 \mathrm{k}_{2} \mathrm{k}_{\mathrm{t}-2} /\left[\left(\mathrm{k}_{2 \mathrm{BN}}+\mathrm{k}_{4 \mathrm{BN}}+\mathrm{k}_{\mathrm{t}}+\mathrm{k}_{\mathrm{esc}}\right)^{2}+\mathrm{k}_{2}\left(\mathrm{k}_{2 \mathrm{BN}}+\mathrm{k}_{4 \mathrm{BN}}+\mathrm{k}_{\mathrm{t}-2 \mathrm{BN}}+\mathrm{k}_{\mathrm{t}-4 \mathrm{BN}}+\mathrm{k}_{\mathrm{esc}}\right)\right]
\end{aligned}
$$

Therefore, as concluded in the text, the efficiency of photoracemization of $(R)-2$ is related to the magnitudes of $\mathrm{k}_{\mathrm{t}}, \mathrm{k}_{2}$, and $\mathrm{k}_{2 \mathrm{BN}}+\mathrm{k}_{4 \mathrm{BN}}+\mathrm{k}_{\mathrm{esc}}$,

\section{Derivation of eq 5 from Scheme 4.}

\section{Scheme 4. A simplified mechanism for in-cage radical-pair B combinations.}

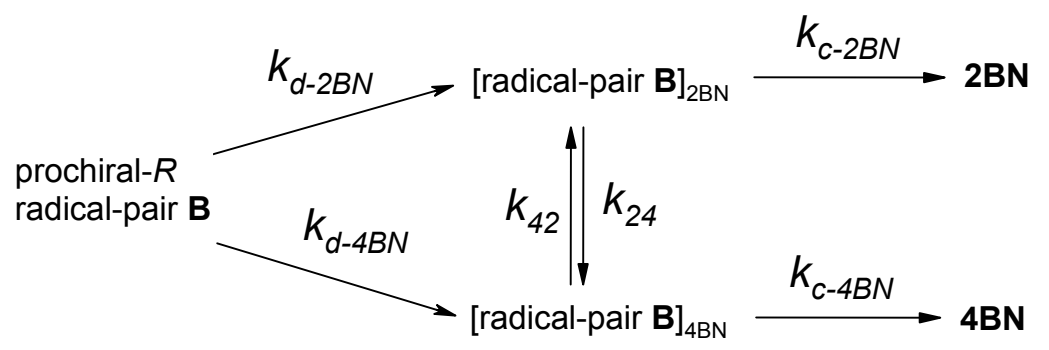

Based on the steady-state approximation for radical-pair $\mathbf{B}$,

$\mathrm{d}[\text { radical-pair } \mathbf{B}]_{2 \mathrm{BN}} / \mathrm{dt}=0$ 
$=\mathrm{k}_{\mathrm{d}-2 \mathrm{BN}}[$ pro- $R$ radical-pair $\mathbf{B}]+\mathrm{k}_{42}[\text { radical-pair } \mathbf{B}]_{4 \mathrm{BN}}-\left(\mathrm{k}_{24}+\mathrm{k}_{\mathrm{c}-2 \mathrm{BN}}\right)[\text { radical-pair } \mathbf{B}]_{2 \mathrm{BN}}$

$\mathrm{d}$ [radical-pair $\mathbf{B}]_{4 \mathrm{BN}} / \mathrm{dt}=0$

$=\mathrm{k}_{\mathrm{d}-4 \mathrm{BN}}[$ pro- $R$ radical-pair $\mathbf{B}]+\mathrm{k}_{24}$ [radical-pair $\left.\mathbf{B}\right]_{2 \mathrm{BN}}-\left(\mathrm{k}_{42}+\mathrm{k}_{\mathrm{c}-4 \mathrm{BN}}\right)[\text { radical-pair } \mathbf{B}]_{4 \mathrm{BN}}$

Therefore,

[radical-pair $\mathbf{B}]_{2 \mathrm{BN}}=\left\{\left[\left(\mathrm{k}_{42}+\mathrm{k}_{\mathrm{c}-4 \mathrm{BN}}\right) \mathrm{k}_{\mathrm{d}-2 \mathrm{BN}}+\mathrm{k}_{42} \mathrm{k}_{\mathrm{d}-4 \mathrm{BN}}\right] /\left[\left(\mathrm{k}_{24}+\mathrm{k}_{\mathrm{c}-2 \mathrm{BN}}\right)\left(\mathrm{k}_{42}+\mathrm{k}_{\mathrm{c}-4 \mathrm{BN}}\right)-\mathrm{k}_{24} \times \mathrm{k}_{42}\right]\right\}$

$\times[$ pro- $R$ radical-pair $\mathbf{B}]$

[radical-pair $\mathbf{B}]_{4 \mathrm{BN}}=\left\{\left[\left(\mathrm{k}_{24}+\mathrm{k}_{\mathrm{c}-2 \mathrm{BN}}\right) \mathrm{k}_{\mathrm{d}-4 \mathrm{BN}}+\mathrm{k}_{24} \mathrm{k}_{\mathrm{d}-2 \mathrm{BN}}\right] /\left[\left(\mathrm{k}_{24}+\mathrm{k}_{\mathrm{c}-2 \mathrm{BN}}\right)\left(\mathrm{k}_{42}+\mathrm{k}_{\mathrm{c}-4 \mathrm{BN}}\right)-\mathrm{k}_{24} \times \mathrm{k}_{42}\right]\right\}$

$\times[$ pro- $R$ radical-pair $\mathbf{B}]$

From eqs S29 and S30,

$\left.(\mathbf{2 B N} / \mathbf{4 B N})_{\text {in-cage }}=\mathrm{k}_{\mathrm{c}-2 \mathrm{BN}}[\text { radical-pair B }]_{2 \mathrm{BN}} /\left(\mathrm{k}_{\mathrm{c}-4 \mathrm{BN}} \text { [radical-pair } \mathbf{B}\right]_{4 \mathrm{BN}}\right)$
$=\mathrm{k}_{\mathrm{c}-2 \mathrm{BN}} / \mathrm{k}_{\mathrm{c}-4 \mathrm{BN}} \times\left[\left(\mathrm{k}_{42}+\mathrm{k}_{\mathrm{c}-4 \mathrm{BN}}\right) \mathrm{k}_{\mathrm{d}-2 \mathrm{BN}}+\mathrm{k}_{42} \mathrm{k}_{\mathrm{d}-4 \mathrm{BN}}\right] /\left[\left(\mathrm{k}_{24}+\mathrm{k}_{\mathrm{c}-2 \mathrm{BN}}\right) \mathrm{k}_{\mathrm{d}-4 \mathrm{BN}}+\mathrm{k}_{24} \mathrm{k}_{\mathrm{d}-2 \mathrm{BN}}\right]$

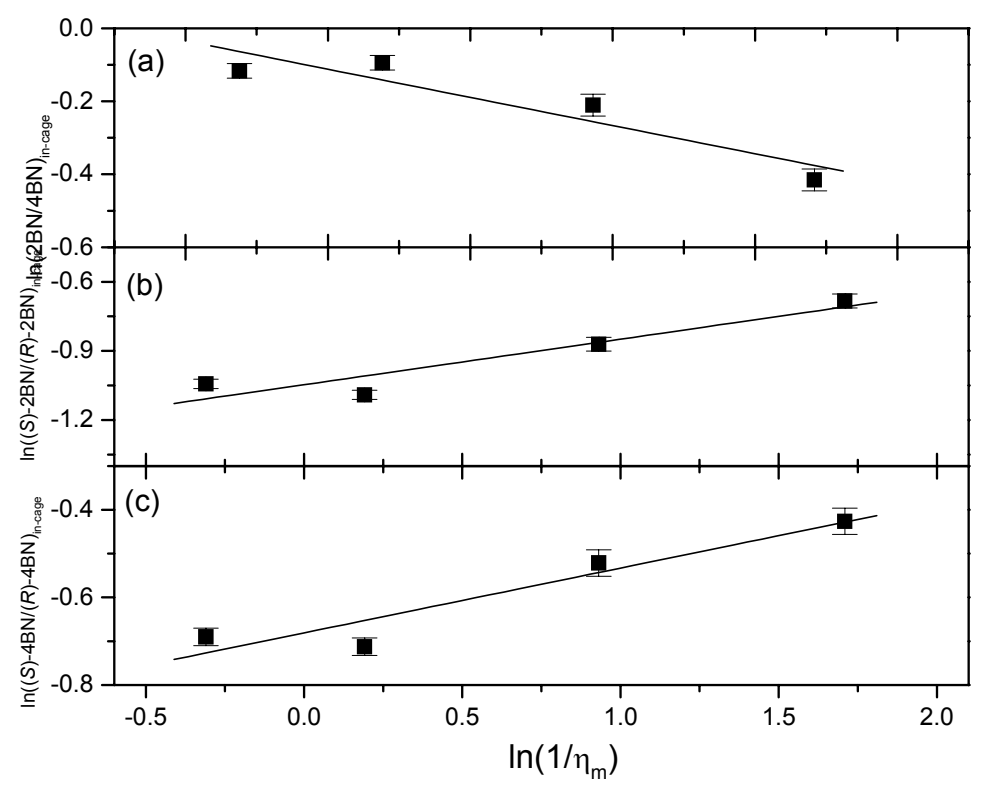

Figure S4. Plots of (a) $\ln (\mathbf{2 B N} / \mathbf{4 B N})_{\text {in-cage, }}$ (b) $\ln ((S)-\mathbf{2 B N} /(R)-\mathbf{2 B N})_{\text {in-cage, }}$ and (c) $\ln ((S)-\mathbf{4 B N} /(R)$ $\mathbf{4 B N})_{\text {in-cage }}$ versus $\ln \left(1 / \eta_{\mathrm{m}}\right)$ for irradiations of $(R)-\mathbf{2}$ in various $n$-alkanes at $23{ }^{\circ} \mathrm{C}$.

From equations S6-S7, 


$$
[(S)] /[(R)]=(1-e e) /(1+e e)
$$

Therefore, $((S)-\mathbf{2 B N} /(R)-\mathbf{2 B N})_{\text {in-cage }}=\left(1-e e_{2 \mathrm{BN} \text {-in-cage }}\right) /\left(1+e e_{2 \mathrm{BN} \text {-in-cage }}\right)$

$$
((S)-\mathbf{4 B N} /(R)-\mathbf{4 B N})_{\text {in-cage }}=\left(1-e e_{4 \mathrm{BN}-\text { in-cage }}\right) /\left(1+e e_{4 \mathrm{BN} \text {-in-cage }}\right)
$$

Table S18. (S)-enantiomer/(R)-enantiomer ratios used in Figure S4.

\begin{tabular}{cccccc}
\hline Medium & $\mathrm{T}\left({ }^{\circ} \mathrm{C}\right)$ & $e e_{2 \mathrm{BN}-\text { in-cage }}$ & $e e_{4 \mathrm{BN}-\text { in-cage }}$ & $((S)-\mathbf{2 B N} /(R)-\mathbf{2 B N})_{\text {in-cage }}$ & $((S)-\mathbf{4 B N} /(R)-\mathbf{B B N})_{\text {in-cage }}$ \\
\hline Hexane & 23 & 32.9 & 21.0 & 0.50 & 0.65 \\
Hexane & 60 & 30.2 & 19.0 & 0.54 & 0.68 \\
Decane & 23 & 41.0 & 25.5 & 0.42 & 0.59 \\
Decane & 60 & 37.4 & 23.4 & 0.46 & 0.62 \\
Tetradecane & 23 & 49.7 & 34.2 & 0.34 & 0.49 \\
Tetradecane & 60 & 41.9 & 29.1 & 0.41 & 0.55 \\
Heptadecane & 23 & 47.9 & 33.2 & 0.35 & 0.50 \\
\hline
\end{tabular}

\section{Geometry optimization of 2 in the ground and excited states: Cartesian coordinates, number of imaginary frequencies, and total energies of optimized structures}

Table S19. Cartesian coordinates of optimized 2-G in its ground state calculated at the RHF/6-31G*

\begin{tabular}{|c|c|c|c|c|c|}
\hline \multirow{2}{*}{$\begin{array}{l}\text { Center } \\
\text { Number }\end{array}$} & \multirow{2}{*}{$\begin{array}{l}\text { Atomic } \\
\text { Number }\end{array}$} & \multirow{2}{*}{$\begin{array}{c}\text { Atomic } \\
\text { Type }\end{array}$} & \multicolumn{3}{|c|}{ Coordinates (Angstroms) } \\
\hline & & & $\mathrm{X}$ & Y & Z \\
\hline 1 & 6 & 0 & -2.451276 & -1.454638 & 0.374613 \\
\hline 2 & 6 & 0 & -3.727659 & -1.665619 & 0.794520 \\
\hline 3 & 6 & 0 & -4.652929 & -0.597627 & 0.828231 \\
\hline 4 & 6 & 0 & -4.273543 & 0.648449 & 0.440148 \\
\hline 5 & 6 & 0 & -2.949528 & 0.902618 & -0.002566 \\
\hline 6 & 6 & 0 & -2.030278 & -0.162847 & -0.033367 \\
\hline 7 & 6 & 0 & -0.693493 & 0.091726 & -0.479782 \\
\hline 8 & 6 & 0 & -0.320953 & 1.342656 & -0.862121 \\
\hline 9 & 6 & 0 & -1.262825 & 2.402211 & -0.828482 \\
\hline 10 & 6 & 0 & -2.535758 & 2.199800 & -0.414768 \\
\hline 11 & 8 & 0 & 0.115321 & -0.991466 & -0.477425 \\
\hline 12 & 6 & 0 & 1.388278 & -0.966384 & -1.084844 \\
\hline 13 & 6 & 0 & 2.439278 & -0.313187 & -0.202168 \\
\hline 14 & 6 & 0 & 1.730992 & -2.421422 & -1.384732 \\
\hline 15 & 6 & 0 & 3.530624 & 0.321672 & -0.780976 \\
\hline 16 & 6 & 0 & 4.528817 & 0.873266 & 0.006129 \\
\hline 17 & 6 & 0 & 4.442625 & 0.799864 & 1.386270 \\
\hline
\end{tabular}
level. 


\begin{tabular}{|c|c|c|c|c|c|}
\hline 18 & 6 & 0 & 3.354378 & 0.171812 & 1.971027 \\
\hline 19 & 6 & 0 & 2.359736 & -0.382882 & 1.182870 \\
\hline 20 & 1 & 0 & -1.746957 & -2.262495 & 0.346201 \\
\hline 21 & 1 & 0 & -4.038842 & -2.647887 & 1.102254 \\
\hline 22 & 1 & 0 & -5.659486 & -0.776969 & 1.162160 \\
\hline 23 & 1 & 0 & -4.975425 & 1.463549 & 0.463576 \\
\hline 24 & 1 & 0 & 0.683468 & 1.552477 & -1.170806 \\
\hline 25 & 1 & 0 & -0.942284 & 3.381573 & -1.136138 \\
\hline 26 & 1 & 0 & -3.243553 & 3.009028 & -0.389807 \\
\hline 27 & 1 & 0 & 1.319671 & -0.422290 & -2.020846 \\
\hline 28 & 1 & 0 & 2.697964 & -2.489138 & -1.870217 \\
\hline 29 & 1 & 0 & 1.768471 & -2.994574 & -0.465468 \\
\hline 30 & 1 & 0 & 0.979571 & -2.855119 & -2.034913 \\
\hline 31 & 1 & 0 & 3.603286 & 0.389327 & -1.853688 \\
\hline 32 & 1 & 0 & 5.366598 & 1.362892 & -0.457715 \\
\hline 33 & 1 & 0 & 5.212937 & 1.231153 & 2.000248 \\
\hline 34 & 1 & 0 & 3.278011 & 0.115287 & 3.042339 \\
\hline 35 & 1 & 0 & 1.515092 & -0.861892 & 1.642213 \\
\hline
\end{tabular}

Number of imaginary frequencies: 0

Total energy: $\mathrm{E}(\mathrm{RHF})=-765.822361450 \quad$ A.U.

\section{Zero-point correction $=0.310564($ Hartree/particle $)$}

Table S20. Cartesian coordinates of optimized $\mathbf{2 - L}$ in its ground state calculated at the RHF/6-31G* level.

\begin{tabular}{|c|c|c|c|c|c|}
\hline \multirow{2}{*}{$\begin{array}{l}\text { Center } \\
\text { Number }\end{array}$} & \multirow{2}{*}{$\begin{array}{l}\text { Atomic } \\
\text { Number }\end{array}$} & \multirow{2}{*}{$\begin{array}{c}\text { Atomic } \\
\text { Type }\end{array}$} & \multicolumn{3}{|c|}{ Coordinates (Angstroms) } \\
\hline & & & $\mathrm{x}$ & $\mathrm{Y}$ & Z \\
\hline 1 & 6 & 0 & 1.092758 & 1.510804 & -0.203573 \\
\hline 2 & 6 & 0 & 1.762097 & 2.679079 & -0.018130 \\
\hline 3 & 6 & 0 & 3.160504 & 2.676897 & 0.198626 \\
\hline 4 & 6 & 0 & 3.848425 & 1.505711 & 0.211089 \\
\hline 5 & 6 & 0 & 3.181551 & 0.267196 & 0.016106 \\
\hline 6 & 6 & 0 & 1.785392 & 0.271074 & -0.178857 \\
\hline 7 & 6 & 0 & 1.120389 & -0.978102 & -0.365838 \\
\hline 8 & 6 & 0 & 1.822563 & -2.140095 & -0.396689 \\
\hline 9 & 6 & 0 & 3.226080 & -2.131552 & -0.212117 \\
\hline 10 & 6 & 0 & 3.887182 & -0.966051 & -0.000711 \\
\hline 11 & 8 & 0 & -0.227388 & -0.983993 & -0.565375 \\
\hline 12 & 6 & 0 & -1.064536 & -0.947101 & 0.583412 \\
\hline 13 & 6 & 0 & -2.353198 & -0.238486 & 0.212572 \\
\hline 14 & 6 & 0 & -1.322744 & -2.362976 & 1.092013 \\
\hline 15 & 6 & 0 & -3.075668 & 0.430305 & 1.193210 \\
\hline 16 & 6 & 0 & -4.279208 & 1.045913 & 0.891437 \\
\hline 17 & 6 & 0 & -4.774225 & 1.003782 & -0.401512 \\
\hline 18 & 6 & 0 & -4.056882 & 0.343978 & -1.386058 \\
\hline 19 & 6 & 0 & -2.854387 & -0.274254 & -1.082911 \\
\hline 20 & 1 & 0 & 0.035423 & 1.514175 & -0.386203 \\
\hline 21 & 1 & 0 & 1.230800 & 3.613621 & -0.042076 \\
\hline 22 & 1 & 0 & 3.676174 & 3.609120 & 0.345477 \\
\hline 23 & 1 & 0 & 4.913235 & 1.500894 & 0.365217 \\
\hline 24 & 1 & 0 & 1.302694 & -3.063690 & -0.570357 \\
\hline 25 & 1 & 0 & 3.761855 & -3.063453 & -0.237329 \\
\hline 26 & 1 & 0 & 4.952792 & -0.959017 & 0.145566 \\
\hline 27 & 1 & 0 & -0.565532 & -0.376597 & 1.358973 \\
\hline
\end{tabular}




\begin{tabular}{rrrrrr}
28 & 1 & 0 & -1.982047 & -2.340406 & 1.952937 \\
29 & 1 & 0 & -1.792436 & -2.957237 & 0.316117 \\
30 & 1 & 0 & -0.395229 & -2.840715 & 1.386393 \\
31 & 1 & 0 & -2.695222 & 0.476104 & 2.199774 \\
32 & 1 & 0 & -4.823844 & 1.561724 & 1.662239 \\
33 & 1 & 0 & -5.706324 & 1.484129 & -0.640227 \\
34 & 1 & 0 & -4.431781 & 0.311420 & -2.393714 \\
35 & 1 & 0 & -2.298011 & -0.774856 & -1.852213 \\
\hline
\end{tabular}

Number of imaginary frequencies: 0

Total energy: $\mathrm{E}(\mathrm{RHF})=-765.818835704$ A.U.

Zero-point correction $=0.310322($ Hartree/particle $)$

Table S21. Cartesian coordinates of optimized 2-G in its first excited singlet state calculated at the RCIS/6-31G* level.

\begin{tabular}{|c|c|c|c|c|c|}
\hline \multirow{2}{*}{$\begin{array}{l}\text { Center } \\
\text { Number }\end{array}$} & \multirow{2}{*}{$\begin{array}{l}\text { Atomic } \\
\text { Number }\end{array}$} & \multirow{2}{*}{$\begin{array}{c}\text { Atomic } \\
\text { Type }\end{array}$} & \multicolumn{3}{|c|}{ Coordinates (Angstroms) } \\
\hline & & & $\mathrm{x}$ & $\mathrm{Y}$ & Z \\
\hline 1 & 6 & 0 & -2.463625 & -1.463755 & 0.368765 \\
\hline 2 & 6 & 0 & -3.773194 & -1.669683 & 0.786225 \\
\hline 3 & 6 & 0 & -4.696951 & -0.603107 & 0.818048 \\
\hline 4 & 6 & 0 & -4.299591 & 0.669801 & 0.425867 \\
\hline 5 & 6 & 0 & -2.990333 & 0.933104 & -0.003681 \\
\hline 6 & 6 & 0 & -2.018098 & -0.193488 & -0.029609 \\
\hline 7 & 6 & 0 & -0.700624 & 0.070721 & -0.457889 \\
\hline 8 & 6 & 0 & -0.308165 & 1.354151 & -0.848433 \\
\hline 9 & 6 & 0 & -1.244125 & 2.405001 & -0.828044 \\
\hline 10 & 6 & 0 & -2.555760 & 2.198814 & -0.416021 \\
\hline 11 & 8 & 0 & 0.128155 & -0.990503 & -0.450945 \\
\hline 12 & 6 & 0 & 1.394843 & -0.960541 & -1.076155 \\
\hline 13 & 6 & 0 & 2.453273 & -0.312039 & -0.199746 \\
\hline 14 & 6 & 0 & 1.733462 & -2.413538 & -1.389270 \\
\hline 15 & 6 & 0 & 3.548632 & 0.309001 & -0.786445 \\
\hline 16 & 6 & 0 & 4.553643 & 0.857338 & -0.006034 \\
\hline 17 & 6 & 0 & 4.470910 & 0.794007 & 1.374930 \\
\hline 18 & 6 & 0 & 3.379176 & 0.179350 & 1.967158 \\
\hline 19 & 6 & 0 & 2.376980 & -0.371801 & 1.185937 \\
\hline 20 & 1 & 0 & -1.775521 & -2.285878 & 0.349534 \\
\hline 21 & 1 & 0 & -4.084122 & -2.652899 & 1.087704 \\
\hline 22 & 1 & 0 & -5.705896 & -0.775578 & 1.143020 \\
\hline 23 & 1 & 0 & -5.007169 & 1.479860 & 0.446853 \\
\hline 24 & 1 & 0 & 0.703797 & 1.557024 & -1.131530 \\
\hline 25 & 1 & 0 & -0.929424 & 3.386277 & -1.131066 \\
\hline 26 & 1 & 0 & -3.248746 & 3.020983 & -0.408393 \\
\hline 27 & 1 & 0 & 1.313138 & -0.410426 & -2.006978 \\
\hline 28 & 1 & 0 & 2.694805 & -2.478033 & -1.886159 \\
\hline 29 & 1 & 0 & 1.781313 & -2.992057 & -0.473942 \\
\hline 30 & 1 & 0 & 0.974589 & -2.842976 & -2.033509 \\
\hline 31 & 1 & 0 & 3.618767 & 0.368424 & -1.859804 \\
\hline 32 & 1 & 0 & 5.394215 & 1.336557 & -0.475613 \\
\hline 33 & 1 & 0 & 5.246769 & 1.222741 & 1.983658 \\
\hline 34 & 1 & 0 & 3.305658 & 0.130501 & 3.039017 \\
\hline 35 & 1 & 0 & 1.529855 & -0.840499 & 1.651146 \\
\hline
\end{tabular}


Number of imaginary frequencies: 0

Total energy: $\mathrm{E}(\mathrm{RHF})=-765.814295502 \quad$ A.U.

\section{Zero-point correction $=0.307572($ Hartree/particle $)$}

Table S22. Cartesian coordinates of optimized 2-L in its first excited singlet state calculated at the RCIS/6-31G* level.

\begin{tabular}{|c|c|c|c|c|c|}
\hline \multirow{2}{*}{$\begin{array}{l}\text { Center } \\
\text { Number }\end{array}$} & \multirow{2}{*}{$\begin{array}{l}\text { Atomic } \\
\text { Number }\end{array}$} & \multirow{2}{*}{$\begin{array}{l}\text { Atomic } \\
\text { Type }\end{array}$} & \multicolumn{3}{|c|}{ Coordinates (Angstroms) } \\
\hline & & & $\mathrm{x}$ & Y & Z \\
\hline 1 & 6 & 0 & 1.107140 & 1.510875 & -0.236719 \\
\hline 2 & 6 & 0 & 1.806215 & 2.696875 & -0.038630 \\
\hline 3 & 6 & 0 & 3.196247 & 2.679909 & 0.201441 \\
\hline 4 & 6 & 0 & 3.888132 & 1.474071 & 0.218131 \\
\hline 5 & 6 & 0 & 3.235116 & 0.249390 & 0.010726 \\
\hline 6 & 6 & 0 & 1.758005 & 0.268131 & -0.191984 \\
\hline 7 & 6 & 0 & 1.103435 & -0.967330 & -0.357708 \\
\hline 8 & 6 & 0 & 1.811810 & -2.165596 & -0.405815 \\
\hline 9 & 6 & 0 & 3.211592 & -2.170342 & -0.247211 \\
\hline 10 & 6 & 0 & 3.902714 & -0.983186 & -0.031936 \\
\hline 11 & 8 & 0 & -0.242437 & -0.993552 & -0.533809 \\
\hline 12 & 6 & 0 & -1.070085 & -0.893078 & 0.620899 \\
\hline 13 & 6 & 0 & -2.367907 & -0.219291 & 0.220346 \\
\hline 14 & 6 & 0 & -1.305659 & -2.278883 & 1.215333 \\
\hline 15 & 6 & 0 & -3.092855 & 0.490069 & 1.170321 \\
\hline 16 & 6 & 0 & -4.304148 & 1.077271 & 0.844745 \\
\hline 17 & 6 & 0 & -4.804765 & 0.965914 & -0.441943 \\
\hline 18 & 6 & 0 & -4.085162 & 0.265543 & -1.396095 \\
\hline 19 & 6 & 0 & -2.874659 & -0.324398 & -1.069074 \\
\hline 20 & 1 & 0 & 0.053532 & 1.541707 & -0.436917 \\
\hline 21 & 1 & 0 & 1.281509 & 3.633427 & -0.071685 \\
\hline 22 & 1 & 0 & 3.723406 & 3.602126 & 0.359826 \\
\hline 23 & 1 & 0 & 4.951121 & 1.471676 & 0.382663 \\
\hline 24 & 1 & 0 & 1.273390 & -3.078117 & -0.574936 \\
\hline 25 & 1 & 0 & 3.744891 & -3.101699 & -0.284189 \\
\hline 26 & 1 & 0 & 4.970137 & -1.002743 & 0.097111 \\
\hline 27 & 1 & 0 & -0.566921 & -0.271554 & 1.352305 \\
\hline 28 & 1 & 0 & -1.955359 & -2.213065 & 2.081267 \\
\hline 29 & 1 & 0 & -1.776464 & -2.923867 & 0.481631 \\
\hline 30 & 1 & 0 & -0.367985 & -2.727277 & 1.525267 \\
\hline 31 & 1 & 0 & -2.708283 & 0.589672 & 2.171308 \\
\hline 32 & 1 & 0 & -4.850400 & 1.625312 & 1.591689 \\
\hline 33 & 1 & 0 & -5.742826 & 1.424401 & -0.699324 \\
\hline 34 & 1 & 0 & -4.464298 & 0.179564 & -2.398920 \\
\hline 35 & 1 & 0 & -2.316945 & -0.856817 & -1.815588 \\
\hline
\end{tabular}

Number of imaginary frequencies: 0

Total energy: $\mathrm{E}(\mathrm{RHF})=-765.810895565$ A.U.

Zero-point correction $=0.307447$ (Hartree/particle $)$ 